\title{
Design and Implementation of Monitoring and Management System for Discrete Manufacturing Process Based on IOT Technology
}

\author{
Qibing Lu ${ }^{*^{\mathrm{a}}}$, Buyun Sheng ${ }^{\mathrm{b}}$ \\ Hubei Digital Manufacturing Key Laboratory, Wuhan \\ University of Technology, Wuhan 430070, China. \\ aluqibing1988@163.com, bshengby@whut.edu.cn
}

\begin{abstract}
For active perception of multi-source information, coupling of heterogeneous information, and effective integration of manufacturing information and knowledge layer, this paper proposed some key technologies and a new implementation framework. The RFID and other sensing technologies were used to acquire the multi-source heterogeneous information. It developed a monitoring and management system for discrete manufacturing process, based on the Internet of Things. The main function modules of the system were characterized. It has been realized active sensing and dynamic optimization of manufacturing process information, and put forward a new mode and way for quantitative analysis and tracking decision optimization of multi-source heterogeneous information.
\end{abstract}

Keywords-Internet of manufacturing things, Multi-source heterogeneous information, Manufacturing process monitoring.

\section{INTRODUCTION}

With the infiltration of Internet of Things (IOT) in manufacturing industry, $R \& D$ process gradually develop towards the mode of "multi-dimension, transparency of widely perception"[1]. Manufacturing system based on IOT, information sensing technology as the driving force, to promote the development of manufacturing industry, to the direction of globalization and informatization. With the continuous emergence of new manufacturing mode, networked manufacturing [2]-[5], cloud manufacturing [6]-[8], service oriented manufacturing [9]-[11] provide the theoretical and technical support to improve the overall operation and management level of the manufacturing industry.

IOT, as the key theory and technology of information technology industry, has been effectively applied in many fields, such as logistics [12] and industrial automation[13]. Nevertheless, there are some exceptions in real-time monitoring of production process. In recent years, the research of some typical discrete manufacturing enterprises shows that, in the process of implementation, the demand and challenges of traditional MES concentrate on such aspects:

(1) The real-time and accuracy of multi-source heterogeneous information collection

With the expanding industrial production and personalized market demand, information are massive and multi-source heterogeneous in discrete manufacturing process. Lack of effectively real-time automatic acquisition and active perception, multi-source heterogeneous information cannot be obtained timely, and integration of data and knowledge layer is not good in manufacturing information system [14][15].

(2) The relevance and effectiveness of perceptual information

The physical space and information space is strongly associated, non deterministic and highly mixed[16]. It brings a great challenge to the discrete manufacturing monitoring common modeling, unified description and system design. It urgent needs a comprehensive analysis and description for the coupling and correlation mechanism of information space and physical space[17].

(3) The real-time dynamic management of manufacturing process information and resources

The production model has changed from mass to "just in time” (JIT), materials tracking and production monitoring are required more timely, flexible and complicated. Due to a lack of production planning and timely feedback of manufacturing process information, it is difficult to conduct effective control and scheduling to the manufacturing process, and real-time monitoring and dynamic optimization of production resources, tasks and quality.

In view of the present situation of backward multi-source heterogeneous information collection method and poor in real time of data, integrating IOT technology, it proposed an open, hierarchical and scalable network framework of monitoring and management system for discrete manufacturing process. The application of IOT technology in discrete manufacturing process, can improve the production process management, timely and effective tracking and control manufacturing resources, shorten the production cycle, improve product quality and production efficiency. 
II THE FRAMEWORK OF MONITORING AND MANAGEMENT SYSTEM FOR DISCRETE MANUFACTURING PROCESS BASED ON IOT TECHNOLOGY

\section{A Section Headings The system architecture of IOT for manufacturing}

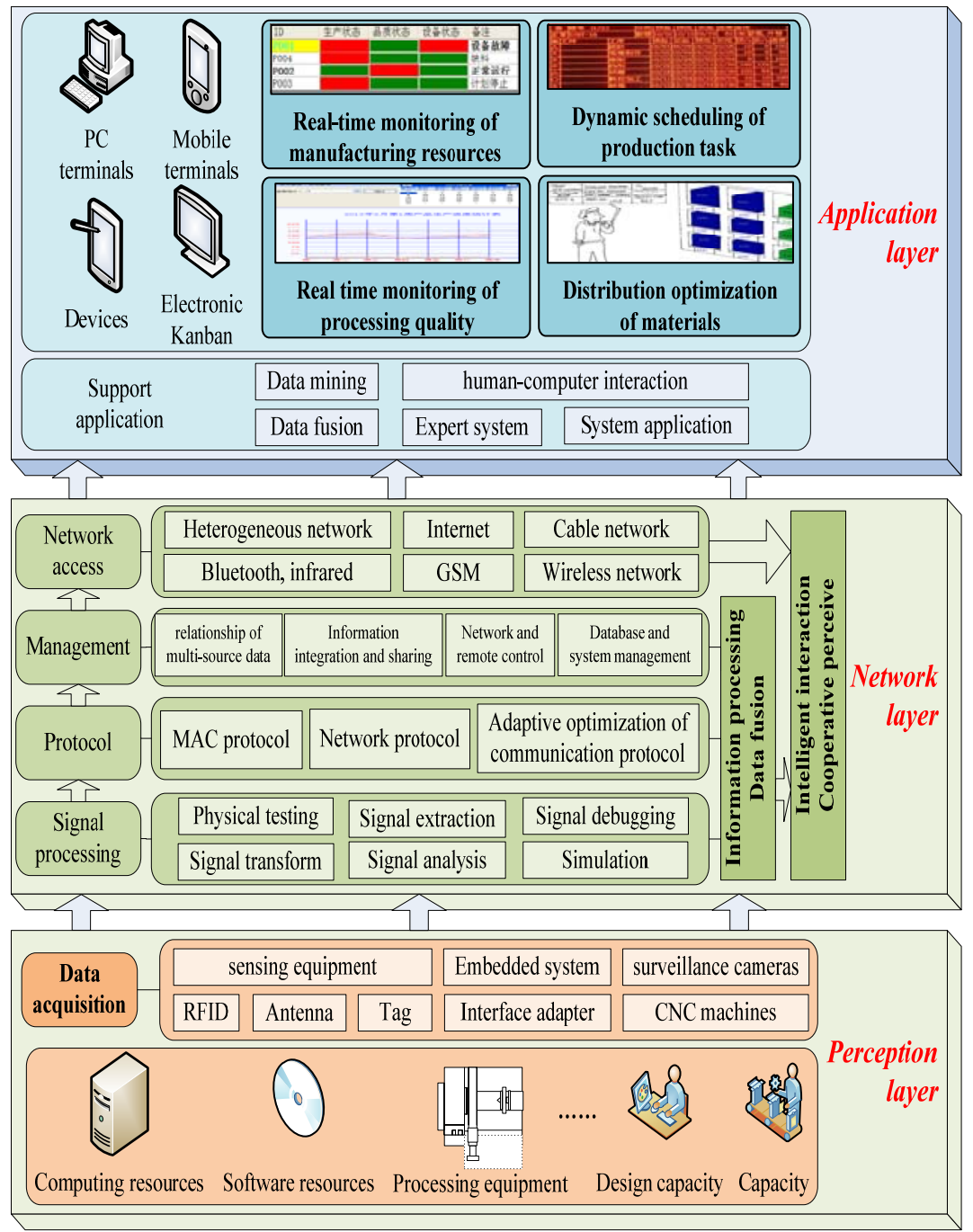

Figure 1. The framework of monitoring and management system for discrete manufacturing process based on IOT technology

IOT for manufacturing mentioned in this paper means that, obtain the required manufacturing process information by RFID, sensors and other sensing device, make exchange and communication of information according to the agreed protocol, to realize intelligent identification, tracking, monitoring and management of manufacturing process. The application of IOT is gradually extensive, with the continuous development of individual application requirements, all kinds of new technology will be gradually integrated into the system of IOT. The system structure design of IOT for manufacturing will determine its technical details, application mode and development trend.

The perception part of IOT is strongly multiple heterogeneous. In order to realize interconnection and interoperability between heterogeneous information, an open, hierarchical and scalable network framework is needed for IOT. In the study of the IOT architecture[18][19], most domestic scholars use the layered network structure, with sensor network layer in the bottom, middle-ware layer of ubiquitous sensor network, application platform layer of ubiquitous sensor network. IOT technology is used in the manufacturing process under the architecture of IOT for manufacturing. All kinds of manufacturing resources connect and communicate with each other. Active perception, dynamic optimization and online monitoring are realized. 


\section{$B$ The system constitution of monitoring and management system for discrete manufacturing process based on IOT technology}

The framework of monitoring and management system for discrete manufacturing process based on IOT technology is designed made up of the perception layer, network layer and application layer. Facing to the physical resources layer in the perception layer, aiming at multi-source manufacturing information, using of RFID and sensor technology and configuration of various types of sensors and wireless network, manufacturing resources information are connected and communicated with each other. In the network layer, through the measurement of a physical quantity, the signal is extracted, debugged, analyzed, then packaged and stored up, integrated according to some certain standards. In the application layer, facing to different users and roles in manufacturing enterprises, by manufacturing data mining, to realize real-time monitoring of manufacturing resource, diagnosis of the processing quality, dynamic scheduling of the production task and the optimization of materials distribution service.

\section{MULTI-SOURCE HETEROGENEOUS INFORMATION SENSING TECHNOLOGY}

In the process of workshop informatization, the first problem to solve is data collection from the production scene. Intelligent sensing technology is mainly to realize the data collection, including sensor technology, embedded technology.

\section{A Manufacturing resource information sensing technology based on macro instruction}

The discrete manufacturing machine is integrated with the upper computer system, by using computer technology, communication technology, and numerical control technology, which aims to realize information interaction and the control and management of processing equipment. Macro method is used for information collection of NC machine with serial communication, by adding specific macros in processing procedures. When the external output command with inlaid user macro program and the NC code with inlaid system variable is executed, CNC machine actively send machine state data to DNC server, the received signal is processed by DNC system. Manufacturing information of CNC machine, collected by macro instruction, include the current program name (component name), start time of processing, end time of processing, speed of main-shaft, feed rate, the cutting tools information.

Inlaid macro instruction in Frank series CNC machine is shown as follows:

POPEN; // Open instruction of external output

DPRNT[O\#4115[40] \#3011[80] \#3012[60]]; // External output instruction

System variables: process name, year, month, day, hour, minute, second.
DPRNT[S\#4119[52]]; // S=3000r/min

DPRNT[F\#4019[52]]; // F=2.5mm/s

DPRNT[T\#4120[30]]; // T03 tool

DPRNT[ \#3011[80] \#3012[60]]; // End time of

instruction

PCLOS; // Close instruction of external output

\section{B Manufacturing information statistics based on RFID technology}

The work-piece equipped with RFID tags read the tag information through the antenna, to obtain the work-piece state and processing information. The process is illustrated in figure 2. The first reading tag information is record as beginning information of processing. The second reading tag information is record as end information of processing. So repeatedly, it is used to obtain process information and the number of defective products.

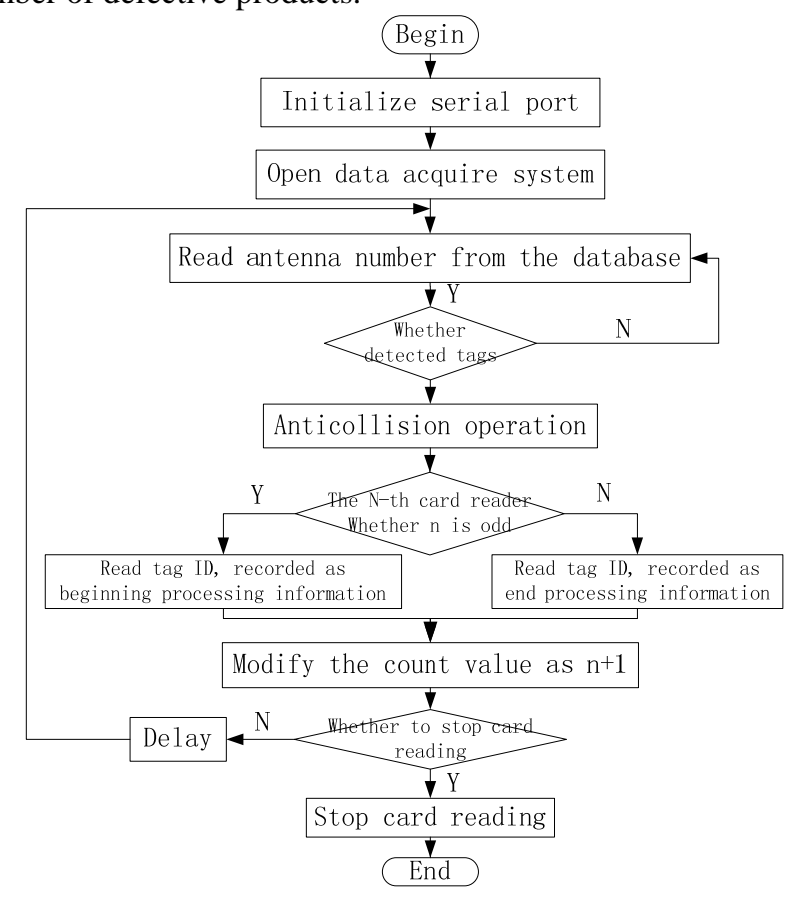

Figure 2. The work-piece tags reading process by RFID

\section{FUNCTION MODULES OF MONITORING AND MANAGEMENT SYSTEM FOR DISCRETE MANUFACTURING PROCESS}

For the collection, processing and tracking management of multi-source heterogeneous information, the function modules of monitoring and management system for discrete manufacturing process consist of four parts:

(1) Management of material and production planning

The unified management of material and production equipment has been done to build equipment resource pool, download the production and materials plan from the ERP system, and generate the production plan and material list of processing station. 
(2) Management of on-site information collection

The system has realized accurate collection the multiple heterogeneous information, including production schedule information, product quality information, equipment state information, and time condition information.

(3) Tracking management of production process

Tracking management of the product life cycle includes implementation of production plan, operation state of machining equipment, product quality tracing, and distribution of materials.

(4) Production statistics and analysis

It includes query of abnormal information in the production process, production execution statistics, and query of manufacturing schedule.

\section{THE DESIGN AND DEVELOPMENT OF MONITORING AND MANAGEMENT SYSTEM FOR DISCRETE MANUFACTURING PROCESS}

In this paper, it has designed and developed monitoring and management system for discrete manufacturing process based on IOT technology, by using Visual Studio 2005 development platform, C\# programming language and SQL Server 2005 system database. It has realized the real-time monitoring and management of discrete manufacturing process. The system modules include monitoring of workshop production status (the current process state of work-piece, the operating condition of processing equipment), management of production process (production orders, BOM, production plan and scheduling, and product quality), management of equipment information (machine, cutting tools, fixture, document), and system management.

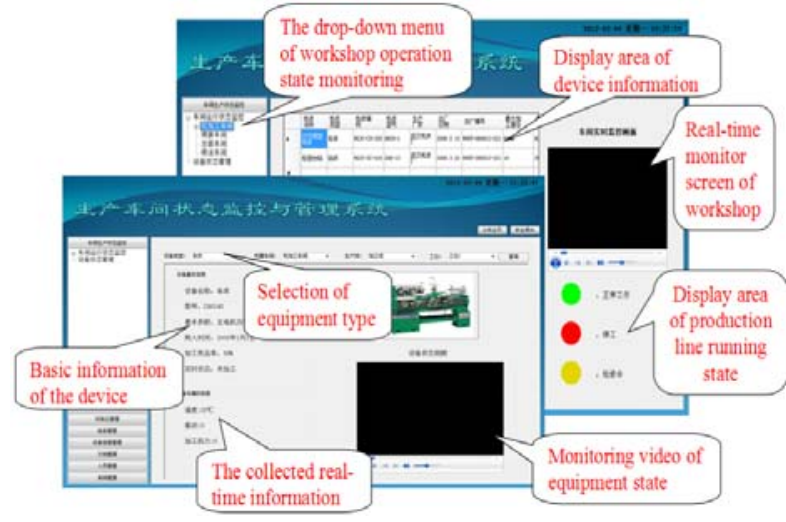

Figure 3. The interface of workshop monitoring and management system

\section{CONCLUSIONS}

High precision and high efficiency developments of modern discrete manufacturing industry set a higher demand on the collection and processing of multi-source heterogeneous information and dynamic monitoring of production process. This paper presents the concept of IOT for manufacturing, has put forward a new mode and way for quantitative analysis and tracking decision optimization of multi-source information in manufacturing process. It presents the framework of monitoring and management system for discrete manufacturing process based on IOT technology. For key technology problems involved in implement process of the system, concrete realization methods are given. It plays a reference role in the field of discrete manufacturing for the application of IOT. Discrete manufacturing systems are developing in the direction of integration, networking and intelligence.

\section{ACKNOWLEDGEMENTS}

This material is based upon work supported by the Fundamental Research Funds for the Central Universities of China (grant no. 2012-IV-037)

\section{CORRESPONDING AUTHOR}

Corresponding author: Qibing Lu. Email: luqibing1988@163.com. Mobile phone: 13429816020.

\section{REFERENCES}

[1] Group of the Strategic Research on the advanced Manufacturing Field: Development Route of Advanced Manufacturing Field in China from Now to 2050 (Science Press, China 2009). (in Chinese)

[2] S. Z. Yang, B. Wu, C. H. Hu, et al.: Network manufacturing and enterprise integration, China Mechanical Engineering, (2000)No.11(1/2), p.45-48. (in Chinese)

[3] Y. K. Lin, P. C. Chang: Reliability-based performance indicator for a manufacturing network with multiple production lines in parallel, Journal of Manufacturing Systems, (2013) No.32, p.147-153.

[4] F. Tao, Y. F. Hu, Z. D. Zhou: Study on manufacturing grid \& its resource service optimal-selection system, International Journal of Advanced Manufacturing Technology, (2008) No.37, p.1022-1041.

[5] Y. S. Fan, D. Z. Zhao, L. Q. Zhang, et al.: Manufacturing grid needs, concept, and architecture, Proceedings of the 2nd International Workshop on Grid and Cooperative Computing(GCC 2003) (Berlin, Germany, 2003) p.653-656.

[6] B. H. Li, L. Zhang, S. L. Wang, et al.: Cloud manufacturing: a new service-oriented networked manufacturing model, Computer Integrated Manufacturing Systems (2010) No.16, p.1-7,16. (in Chinese).

[7] X. Xun: From cloud computing to cloud manufacturing, Robotics and Computer Integrated Manufacturing (2012) No.28, p.75-86.

[8] B. Q. Huang, C. H. Li, C. Yin, et al.: Cloud manufacturing service platform for small- and medium-sized enterprises, The International Journal of Advanced Manufacturing Technology (2013) No.65, p.1261-1272.

[9] F. Tao, L. Zhang, V. C. Venkatesh, et al.: Cloud manufacturing: a computing and service-oriented manufacturing model, Proceedings of the Institution of Mechanical Engineers. Part B, Journal of Engineering Manufacture (2011) No.225, p.1969-1976.

[10] L. Y. Sun, G. Li, Z. B. Jiang, et al.: Service-embedded manufacturing: advanced manufacturing paradigm in 21st century, China Mechanical Engineering (2007) No.18, p.2307-2312. (in Chinese)

[11]H. Li, Y. J. Ji, G. N. Qi, et al.: Connotation, theory and key technologies system on the fusion of manufacturing and services, Computer Integrated Manufacturing Systems (2010) No.16, p.2521-2529. (in Chinese)

[12]B. Li, W. F. Li: Container terminal logistics systems collaborative scheduling based on multi-agent systems, Computer Integrated Manufacturing Systems (2011) No.17, p.2502-2513. (in Chinese) 
[13]N. Li, M. Liu, J. W. Yan: Framework for industrial internet of things oriented to steel continuous casting plant MRO, Computer Integrated Manufacturing Systems (2011) No.17, p.413-418. (in Chinese)

[14] Q. Xu, Q. Robin: Heterogeneous knowledge syntheses for manufacturing information systems, Computer Integrated Manufacturing Systems (2004) No.10, p.732-736,750. (in Chinese)

[15] L. Tong, P. Yan, F. Liu: Service-oriented information integration system for workshop manufacturing process, Computer Integrated Manufacturing Systems (2010) No.16, p.340-348. (in Chinese)

[16] L. N. Zeng, J. W. Ding, J. Zhao, et al.: Detecting and modeling for associations between high-dimension condition monitoring data of complex equipment based on mutual information, Computer Integrated Manufacturing Systems (2013) No.19, p.3017-3025. (in Chinese)

[17] J. Ma, T. Y. Xiao, W. H. Fan: Information model of correlation for district collaborative design, Computer Integrated Manufacturing Systems (2009) No.15, p.1484-1492. (in Chinese)
[18] X. F. Yao, M. Yu, Y. Chen, et al.: Connotation, architecture and key technologies of internet of manufacturing things, Computer Integrated Manufacturing Systems (2014) No.20, p.1-10. (in Chinese)

[19] H. M. Chen, L. Cui, K. B. Xie, et al.: A comparative study on architectures and implementation methodologies of internet of things, Computer Integrated Manufacturing Systems (2013) No.36, p.168-188. (in Chinese)

[20] Q. Yin , L. Z. Wang, Q. M. Tian: Algorithm of mining association rules with weighted items based on probability, Computer Applications (2005) Vol.25, No.4, p.805-807.

[21] D. C. Huang, H. D. Guo: Hybrid genetic algorithm for solving job scheduling problem on non-identical parallel machines based on JIT technique, Computer Integrated Manufacturing Systems (2004) No.10, p.298-302.

(in

Chinese) 